\title{
Development of a haptic glove to use a master-slave rehabilitation robot hand system
}

\author{
Takashi KOMEDA*, Shinichi UCHINO*, Yoshiyuki TAKAHASHI, Mario ELASYEH**, \\ Yukio KAWAKAMI, Hiroyuki KOYAMA* and Shin-ichiro YAMAMOTO* \\ * School of Systems Engineering \\ Shibaura Institute of Technology \\ Fukasaku 307, Minuma-ku, Saitama, 337-8570, Japan \\ (E-mail: komeda@se.shibaura-it.ac.jp) \\ ** Department of Machine Design \\ Royal Institute of Technology \\ S100-44, Stockholm, Sweden
}

\begin{abstract}
We have developed a haptic glove system to use rehabilitation field, e.g. rehabilitation robotics system. This glove has angle sensors to detect each finger position and small balloons inside of the glove to present a force to the user who wear the glove by pressurized air. This glove makes it possible to give angle information of each finger to slave hand system, and also to present the force which is detected the slave hand to the glove user. To control this force feedback system, we use PWM control method to show the quantity of the force. Force affecting the user is updated by a frequency of $10 \mathrm{~Hz}$ and has several steps resolution. Total weight of this glove with haptic feedback capabilities is less than 200 grams. We tried to use this glove as a master-slave hand system, we could feel the slave hand force though haptic glove like a real force.
\end{abstract}

\section{KEY WORDS}

Haptic device, Force feedback, Robot hand, PWM control

\section{INTRODUCTION}

It is exhausting, both physically and emotionally, to assist physically handicapped persons in 24 hours. As an one of solution to solve this problem, an assistive mobile system called AMOS (Assistive MObile robot System) has been developed to help alleviate this burden [1]. The purpose of AMOS is to pick up and transport daily use objects, placing them in a designated indoor location semi-autonomously. The target objects identified by AMOS are items used on a daily basis, and they have different sizes and shapes. However, it is difficult to recognize a target object, to measure its location using image processing in real-time and to manipulate using robotic hand automatically. Therefore, we are developing and propose a user interface system which allows the user to intervene in the robotic hand control. Almost users of this robot system may not have knowledge about computer and robot, it is required simple and easy user interface. We are trying to develop 


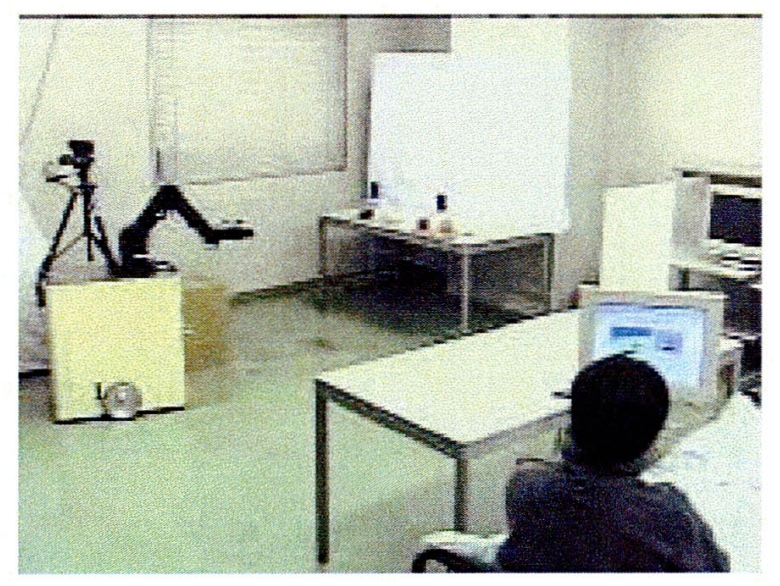

Figure 1 Assistive Mobile Robot System

a master-slave robotic hand system. The user wears the master glove and tries to grasp the target object, the slave hand moves simultaneously with the master. The robot hand as a slave is guided to the target object using image processing. The user can select the object and decide how to grasp it by her/him with force feedback.

The aim of this research is to provide a simple user interface for handling the target object, which has different sizes and shapes. In this paper, we describe the master glove system and its force feedback experiments using pressurized air.

\section{Master Slave Hand System}

The image of the master slave hand system shows in Fig.2. This system is composed of a haptic glove on master side (master hand), and a robot hand with finger component on slave side (slave hand). Slave hand follows master hand, and grasps the target object by user put on master hand. The force when the slave hand holds the object can be fed back to the master hand and the master hand expresses the sense of force that corresponds to the force of the holding to the user by swelling the balloon inside the glove.

Haptic glove is composed of glove, flex sensors, balloons, air valves and a micro controller. It goes for the micro controller to data processing of the finger angle with the flex sensor, and control of the valve for expansion and shrinkage the balloons to present the force. These data are sending to personal computer by CAN (Controller Area Network). The position sensors are attached on the thumb, index and middle finger. They can measure the angle of each finger of the user.

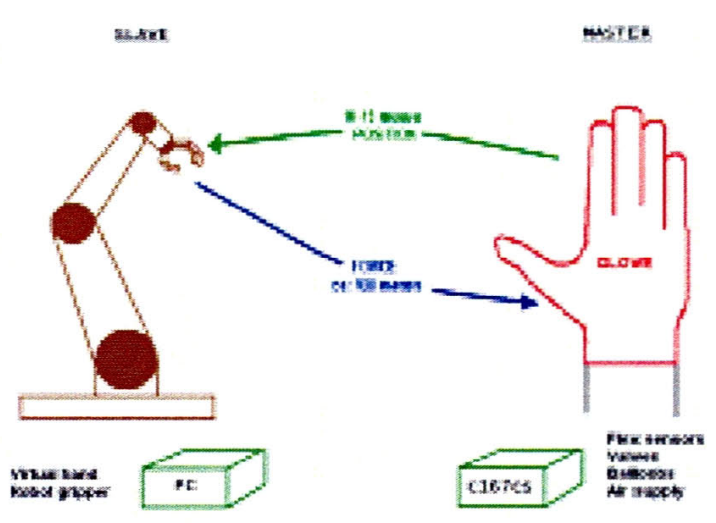

Fig.2 Image of Master Slave Hand

The force when slave hand grasps a target object, it is possible to express it as a sense of force by swelling the balloon which was installed in the thumb, the index finger, the long finger and push to each abdomen by compressed air (Fig. 3).

Fig.4 shows external view of the slave glove and the allocation of the sensors. The position sensor is attached on the surface of the glove. It is a flexible material and the resistance is changed due to the angle of the sensor.

Intake and exhaust pneumatic valves (Fest co., high speed switching valve) are controlled by the micro controller using regulated inner pressure of the balloons. Then, the user can feel the grasping force as he/she handles the object directly. The micro controller (SIEMENS Infenion Co., C167) communicates with slave finger controller (PC) sending position data of the master finger and receive the force data to display on the slave finger using CAN interface.[2]
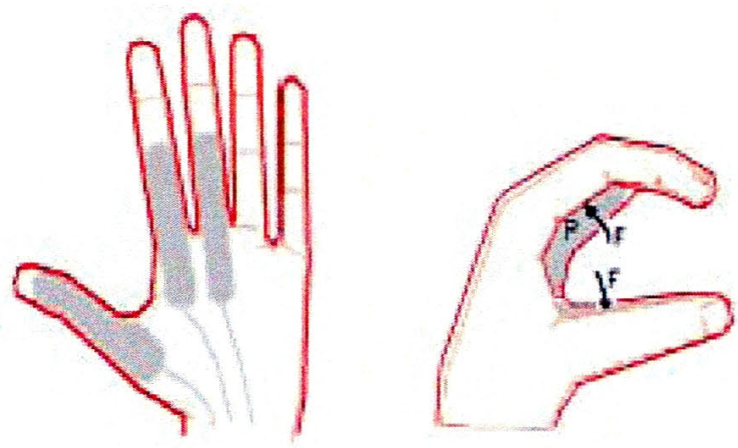

Figure 3. Balloons allocation 


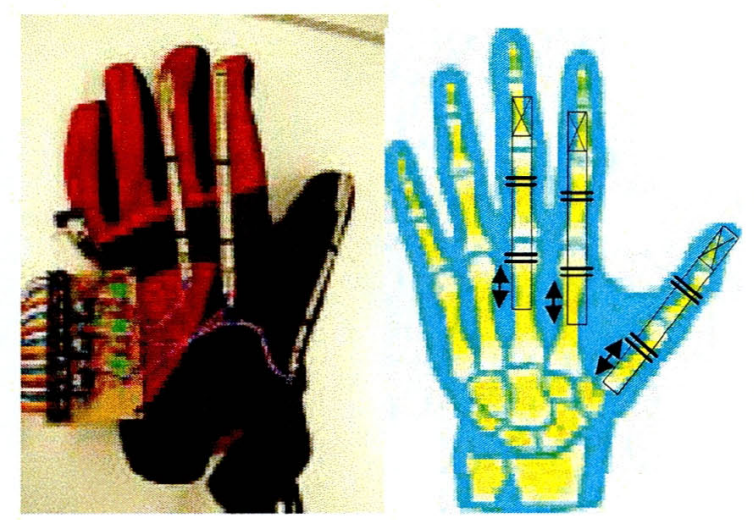

Figure 4 External view and sensor allocation

\section{Experiments of force feedback}

Main objectives of these experiments are to determine the balloons state during intake and exhaust of air. Particularly significant for this system is the ability of the master glove to provide sufficient force feedback. Another important objective is to conclude the reaction time or response time of the balloons inflations and deflations. They are essential in order to estimate the total delay time obtained when inflating and deflating a balloon. We decided to use PWM control to preset force with several steps resolution, because it is easy to change the level of force by computer to change duty ratio. [3]

Outline of Experimental apparatus is shown in Figure 5. A pressure gage sensor is used to determine maximum inflation response time of one balloon, affected only by the pressure inside the balloon. It was attached to one balloon outlet and at the other outlet a coupling connecting pressure-supply and exhaust.

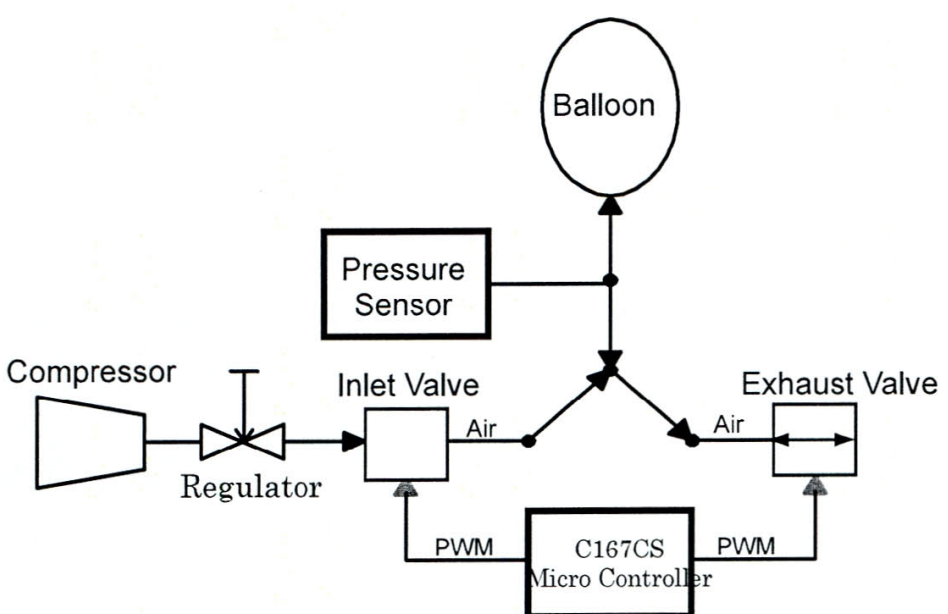

Figure 5. Experimental setup

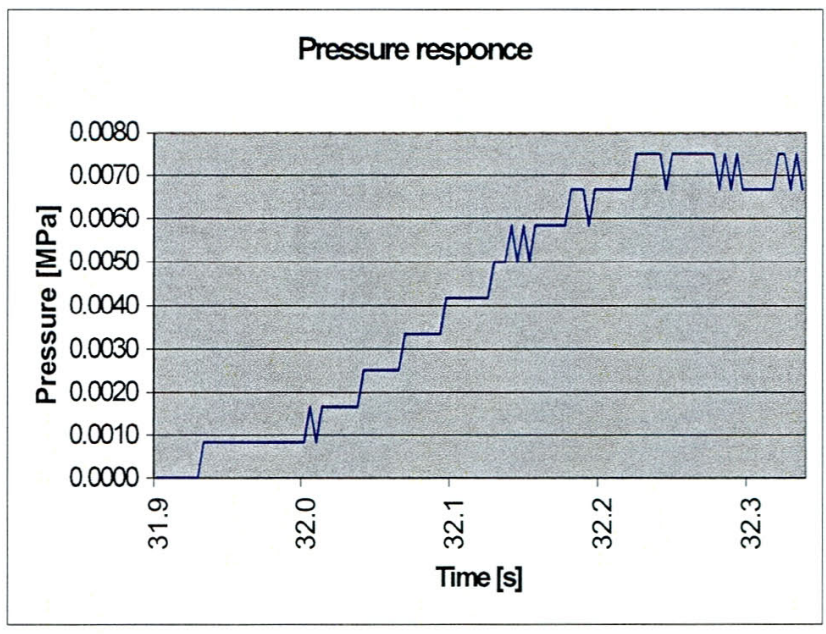

Figure 6 Pressure change at $100 \%$ duty

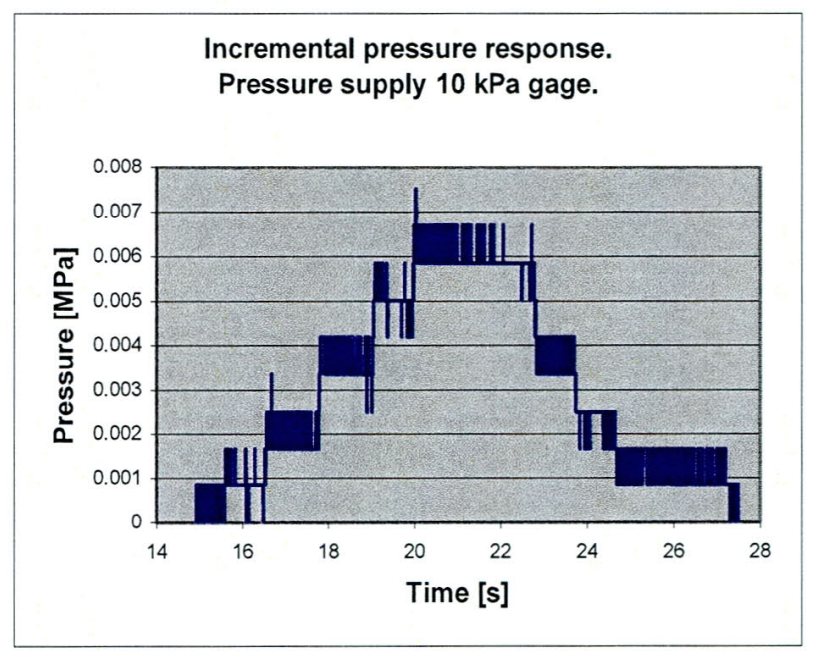

Figure 7 Pressure according to the duty ratio

The micro controller on the master side controls both the suction valve and the exhaust valve. In the program, either the suction valve or the exhaust valve can be operated simultaneously. Air in the balloon is discharged into an atmospheric inside by the exhaust valve's pressure in the balloon increasing when the supply pressure from the compressor connects with the suction valve.

Exhaust of the air pressure to the balloon was assumed to do the opening and shutting time of the valve by the PWM method. To fill the demanded PWM cycle, the supply pressure was calculated using following equation. [2]

$P_{u}=\frac{V_{b} \cdot d_{A I R}}{S_{e m} \cdot T_{O N} \cdot \sqrt{\frac{k}{R \cdot \theta_{a}}\left(\frac{2}{k+1}\right)^{\frac{k+1}{k-1}}}}$ 
Here,

$\mathrm{V}_{\mathrm{b}}$ : Estimated volume a balloon

$\mathrm{d}_{\mathrm{air}}$ : Density of air

$\mathrm{k}$ : Isentropic constant of air $(=1.4)$

$\mathrm{R}$; Gas constant

$\mathrm{S}_{\mathrm{em}}$ : Valve orifice area

Figure 6 shows one example of the experiment result of recording a pressure increase when supplying it from pressure value 0 to the maximum by $100 \%$ with the duty of PWM. It rises to about $7 \mathrm{kPa}$ though it doesn't reach $10 \mathrm{kPa}$ though $250 \mathrm{~ms}$ passes. It is thought that time was required from no rise of pressure immediately after the supply to the swelling of the balloon.

Secondly, when change duty ratio of PWM, we experimented whether the difference of pressure was able to expressed. Six states were set by setting the state of pressure to six stages including the state not pressured, we increased the duty ratio each $20 \%$ from $0 \%$. Result is shown in Figure 7 . We confirmed internal pressure had changed according to the duty. Therefore, it is possible to present the level of force using these balloons and PWM control. A smooth result was not obtained in Fig.7, the reason is only the resolution of the pressure sensor was not enough.

From these results, we checked whether the sense of force is able to recognize by human. It is interview experiments and number of subjects is six. There are two processes to present the force. One is swelling process, it means to give force stronger than previous status (Ex1). The other one is shrinking process, to give force weaker (Ex2). It is different process, but, if system present same duty ratio of PWM, it must be feel same force. Fig. 8 shows correct answer rate of each subject. Highest score is over $80 \%$, it is not $100 \%$. Then, it is difficult to present the force precisely using this method. Especially, shrinking process is lower than swelling process and the lowest correct answer rate is $0 \%$. The human sense of force is not so correct and it is better to present the force using the swelling process even there is an individual variation.

\section{Conclusion}

The master glove system with haptic force feedback has been developed. The master glove can sense finger angle and provide virtual haptic force on each finger using inside balloons and PWM control. We confirmed that the system can present the different force level using this method. However, it was difficult to be feel the different level of force to human. We will try to change PWM frequency and present method to aim $100 \%$ correct answer rate, and to improve for practical use.

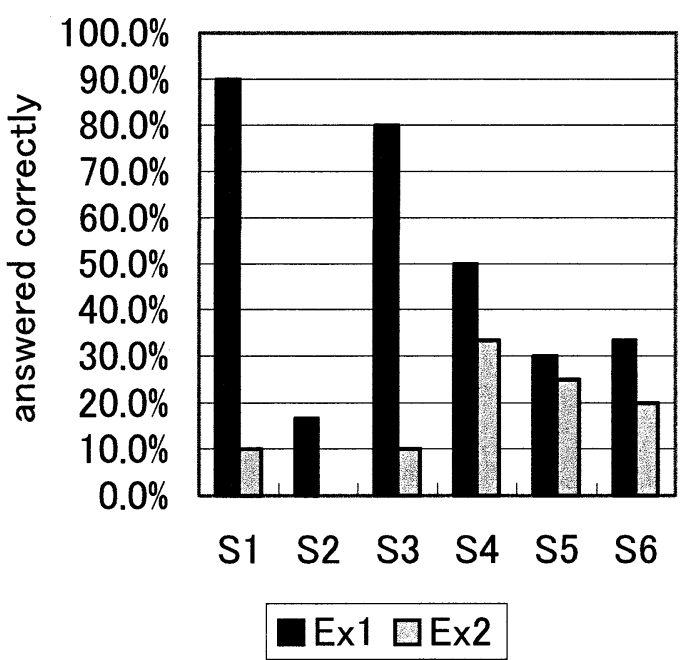

Figure 8 Correct answer rate

\section{References}

1. Y. Takahashi, T. Komeda and H. Koyama, " Development of the Assistive Mobile Robot System: AMOS - To Aid in the Daily Life for Physically Handicapped", Advanced Robotics, Vol.18, No.5, pp.473-496, 2004

2. Mario Elsayeh "Development of haptic glove and interacting virtual graphic user interface", Master of Science Thesis Stockholm (2003)

3. Yukio Kawakami et.al. "Pulse-Width-Modulation control of a pneumatic actuator", $4^{\text {th }}$ International Conference on Control, Automation, Robotics and Vision (1996) 